\title{
Remarks on New Properties of Preinvex Functions
}

\author{
Zaiyun Peng \\ Institute of Mathematics, College of Science \\ Chongqing JiaoTong University \\ Xue Fu Da Dao Street, Nan Ping, Chongqing 400074, China \\ E-mail: pengzaiyun@126.com. \\ Yawei Liu \\ Institute of Mathematics, College of Science, Chongqing JiaoTong University \\ Chongqing 400074, China \\ X. J. Long \\ College of Mathematics and Statistics, ChongqingTechnology and Business University \\ Chongqing 400067, China
}

\begin{abstract}
A new type of generalized convex functions, termed preinvex functions, is further discussed in this paper. A new result that if a preinvex function satisfies intermediate-point semistrict preinvexity then it is also a semistrictly preinvex function is obtained. Furthermore, another simplified proof for a criterion of preinvex functions under weaker conditions and an impotant application of preinvex functions are given.
\end{abstract}

Keywords: Preinvex functions, Semistrictly preinvex functions, Lower semicontinuity, Applications

$\operatorname{MSC}(2000): 90 C 26,26 B 25$

CLC number: 0174.13

\section{Introduction}

Convexity and generalized convexity play a central role in mathematical economics and optimization theories. Therefore, the research on convexity or generalized convexity becomes one of the most important aspects in mathematical programming. A significant generalization of convex functions termed preinvex functions was introduced by (Weir and Mond,1988). Yang and Li obtained some properties of preinvex function in (Yang and Li, 2001). Yang also discussed the relationships among convexity, semistrict convexity and strict convexity in (Yang,1994). In this paper, preinvex functions is further discussed in this paper. A new result that if a preinvex function satisfies intermediate-point semistrict preinvexity then it is also a semistrictly preinvex function is obtained. Furthermore, another simplified proof for a criterion of preinvex functions under weaker conditions and an impotant application of preinvex functions are given.

Now we recall some definitions .

Definition 1.1 (Weir,1998,p.29) A set $K \subseteq R^{n}$ is said to be invex if there exist a vector function $\eta: R^{n} \times R^{n} \rightarrow R^{n}$, such that

$\forall x, y \in K, \forall \lambda \in[0,1] \Rightarrow y+\lambda \eta(x, y) \in K$

Definition 1.2 (Yang,2001) Let $K \subseteq R^{n}$ be an invex set with respect to $\eta: R^{n} \times R^{n} \rightarrow R^{n}$. Let $f: K \rightarrow R$. We say that $f$ is preinvex on $K$, if $\forall x, y \in K, \lambda \in[0,1]$, 
$f(y+\lambda \eta(x, y)) \leq \lambda f(x)+(1-\lambda) f(y)$.

Definition 1.3 (Yang,2001) Let $K \subseteq R^{n}$ be an invex set with respect to $\eta: R^{n} \times R^{n} \rightarrow R^{n}$. Let $f: K \rightarrow R$. We say that $f$ is semistrictly preinvex on $K$, if $\forall \lambda \in(0,1) \forall x, y \in K, f(x) \neq f(y)$, $f(y+\lambda \eta(x, y))<\lambda f(x)+(1-\lambda) f(y)$.

Condition C. Let $\eta: R^{n} \times R^{n} \rightarrow R^{n}$. We say that the vector-valued function $\eta$ satisfies Condition $\mathrm{C}$ if for any $x, y \in K$ any $\lambda \in(0,1)$,

$\eta(y, y+\lambda \eta(x, y))=-\lambda \eta(x, y)$,

$\eta(x, y+\lambda \eta(x, y))=(1-\lambda) \eta(x, y)$.

Example1. Let $\eta(x, y)=\left\{\begin{array}{l}x-y,(x \geq 0, y \geq 0) \\ x-y,(x<0, y<0) \\ -2-y,(x>0, y \leq 0) \\ 2-y,(x \leq 0, y>0)\end{array}\right.$

By Example 2.2 in (Yang, 2003), we know that $\eta$ satisfies the Condition C. Another example that $\eta$ satisfies the Condition C may refer Example 2.4 in (Yang, 1995, p.901).

\section{Main Results}

In (Yang,2001, p.256), the authors have discussed the preinvex functions and semistrictly preinvex functions.In this section, we will discuss the properties of preinvex functions. Firstly, we give the following basic lemma.

Lemma 2.1 Let $f$ be a lower semicontinous and satisfy $f(y+\eta(x, y)) \leq f(x)(\forall x, y \in K)$, $\eta: R^{n} \times R^{n} \rightarrow R^{n}$ satisfies Condition C. If for every pair $x, y \in K$, there exist $\lambda \in(0,1)$ such that inequality

$$
f(y+\lambda \eta(x, y)) \leq \lambda f(x)+(1-\lambda) f(y)
$$

then the set $A(x, y)=\{\alpha \in[0,1] \mid f(y+\alpha \eta(x, y)) \leq \alpha f(x)+(1-\alpha) f(y)\}, \forall x, y \in K$ is dense in the interval $[0,1]$.

Proof . Given $x, y \in K, x \neq y$, suppose that $A(x, y)$ is not dense in $[0,1]$. Then , there exist $\bar{\alpha} \in(0,1)$ and $\varepsilon>0$ such that

$$
A(x, y) \cap(\bar{\alpha}-\varepsilon, \bar{\alpha}+\varepsilon)=\phi
$$

For $f(y+\eta(x, y)) \leq f(x)(\forall x, y \in K)$, then $0 \in A(x, y)$, and $1 \in A(x, y)$.

Define

$$
\begin{aligned}
& u=\sup \{\alpha \in A(x, y): \alpha<\bar{\alpha}\} \\
& v=\inf \{\alpha \in A(x, y): \alpha>\bar{\alpha}\}
\end{aligned}
$$

Let $u_{1} \leq u_{2} \leq \ldots \leq u_{k} \leq \ldots$, such that $u_{k} \rightarrow u$ as $k \rightarrow+\infty$, and $u_{k} \in A(x, y)$ for each $k$, then

$$
\begin{aligned}
& f\left(y+u_{k} \eta(x, y)\right) \leq u_{k} f(x)+\left(1-u_{k}\right) f(y) \\
& \liminf _{k \rightarrow \infty} f\left(y+u_{k} \eta(x, y)\right) \leq \lim _{k \rightarrow \infty} \inf \left[u_{k} f(x)+\left(1-u_{k}\right) f(y)\right]
\end{aligned}
$$

Since $f$ is a lower semicontinous, we have

$$
f(y+u \eta(x, y)) \leq \liminf _{k \rightarrow \infty} f\left(y+u_{k} \eta(x, y)\right)
$$

Form (2.2) and (2.3), we obtain $f(y+u \eta(x, y)) \leq u f(x)+(1-u) f(y)$.Hence $u \in A(x, y)$.

Similarly, we get $v \in A(x, y)$. 
Then, by the definitions of $u$ and $v$, we obtain $0 \leq u<\bar{\alpha}<v \leq 1$, and

$$
\alpha u+(1-\alpha) v \notin A(x, y), \forall \alpha \in(0,1)
$$

Now denote $x_{u}=y+u \eta(x, y), y_{v}=y+v \eta(x, y)$

Then under inequality (2.1), there exist a $\lambda \in(0,1)$ such that

$$
f\left(y_{v}+\lambda \eta\left(x_{\mathrm{u}}, y_{v}\right)\right) \leq \lambda f\left(x_{\mathrm{u}}\right)+(1-\lambda) f\left(y_{v}\right)
$$

From Condition $\mathrm{C}$, we have

$$
\begin{aligned}
& y_{v}+\lambda \eta\left(x_{\mathrm{u}}, y_{v}\right)=y+v \eta(x, y)+\lambda \eta(y+u \eta(x, y), y+v \eta(x, y)) \\
= & y+v \eta(x, y)+\lambda \eta(y+u \eta(x, y), y+u \eta(x, y)-(u-v) \eta(x, y)) \\
= & y+[\lambda u+(1-\lambda) v] \eta(x, y)
\end{aligned}
$$

Hence, by (2.5) and $u, v \in A(x, y)$, we obtain

$$
\begin{aligned}
& f(y+[\lambda u+(1-\lambda) v] \eta(x, y))=f\left(y_{v}+\lambda \eta\left(x_{u}, y_{v}\right)\right) \\
& \leq \lambda f\left(x_{u}\right)+(1-\lambda) f\left(y_{v}\right) \leq[\lambda u+(1-\lambda) v] f(x)+[1-(\lambda u+(1-\lambda) v)] f(y)
\end{aligned}
$$

Which implies $\lambda u+(1-\lambda) v \in A(x, y)$, and contradicts (2.4). Thus this proves Lemma2.1.

Theorem 2.2 Let $f$ be a lower semicontinous and satisfy $f(y+\eta(x, y)) \leq f(x)(\forall x, y \in K)$, $\eta: R^{n} \times R^{n} \rightarrow R^{n}$ satisfies Condition C. If for every pair $x, y \in K$, there exist $\lambda \in(0,1)$ such that inequality (2.1) holds, then $f$ is a preinvex function on $K$.

Proof. By Lemma2.1, $\forall x, y \in K, A(x, y)$ is dense in the interval [0,1].

Then, $\forall \bar{\alpha} \in(0,1), \exists\left\{\alpha_{n}\right\} \subseteq(0,1) \cap A(x, y)$, such that $\alpha_{n} \rightarrow \bar{\alpha}$ as $n \rightarrow \infty$.

Since $f\left(y+\alpha_{n} \eta(x, y)\right) \leq \alpha_{n} f(x)+\left(1-\alpha_{n}\right) f(y)$, then

$$
\liminf _{k \rightarrow \infty} f\left(y+\alpha_{k} \eta(x, y)\right) \leq \liminf _{k \rightarrow \infty}\left[\alpha_{k} f(x)+\left(1-\alpha_{k}\right) f(y)\right]
$$

Since $f$ is a lower semicontinous, we have

$$
f(y+\bar{\alpha} \eta(x, y)) \leq \liminf _{k \rightarrow \infty} f\left(y+\alpha_{k} \eta(x, y)\right)
$$

Thus

$$
f(y+\bar{\alpha} \eta(x, y)) \leq \bar{\alpha} f(x)+(1-\bar{\alpha}) f(y)
$$

From Condition $f(y+\eta(x, y)) \leq f(x)(\forall x, y \in K)$, when $\lambda=0,1$ we also have

$$
f(y+\lambda \eta(x, y)) \leq \lambda f(x)+(1-\lambda) f(y)
$$

So $f$ is a preinvex function on $K$. This completes the proof.

Remark 2.1 A criterion of preinvex functions is given by theorem 2.2. Comparing with (Yang,2001), we give another simplified proof for the result.

Theorem 2.3 Let $K \subseteq R^{n}$ be an invex set with respect to $\eta: R^{n} \times R^{n} \rightarrow R^{n}$ which satisfies Condition C, and $f: K \rightarrow R$ be a preinvex function for the same $\eta$. If there exists an $\alpha \in(0,1)$, such that for all $x, y \in K, f(x) \neq f(y)$ implies

$$
f(y+\alpha \eta(x, y))<\alpha f(x)+(1-\alpha) f(y),
$$

Then $f$ is semistrictly preinvex on $K$ with respect to $\eta$.

Proof. On the contrary, we assume that there exist $x, y \in K, \lambda \in(0,1)$ such that $f(x) \neq f(y)$ and 


$$
f(y+\lambda \eta(x, y)) \geq \lambda f(x)+(1-\lambda) f(y) .
$$

Without loss of generality, we assume the $f(x)<f(y)$, let $z=y+\lambda \eta(x, y)$.

Then, inequality (2.9) implies

$$
f(z) \geq \lambda f(x)+(1-\lambda) f(y)>f(x) .
$$

Since $f$ is preinvex function, we have

$$
f(z) \leq \lambda f(x)+(1-\lambda) f(y) .
$$

Which together with $(2.10)$, leads to

$$
f(x)<f(z)=\lambda f(x)+(1-\lambda) f(y) .
$$

Let

$$
\begin{gathered}
z_{1}=z+\alpha \eta(x, z) \\
z_{2}=z+\alpha \eta\left(z_{1}, z\right) \\
\ldots \\
z_{k}=z+\alpha \eta\left(z_{k-1}, z\right), \forall k \in N
\end{gathered}
$$

According to (2.11) and (2.8), we have

$$
\begin{gathered}
f\left(z_{1}\right)=f(z+\alpha \eta(x, z))<f(z) . \\
f\left(z_{2}\right)=f\left(z+\alpha \eta\left(z_{1}, z\right)\right)<f(z) . \\
\ldots \\
f\left(z_{k}\right)=f\left(z+\alpha \eta\left(z_{k-1}, z\right)\right)<f(z) .
\end{gathered}
$$

From Condition C, we have

$$
z_{k}=z+\alpha^{k} \eta(x, z)=y+\left[\lambda+\alpha^{k}(1-\lambda)\right] \eta(x, y)
$$

Let $k_{1} \in N$ be such that

$$
\alpha^{\mathrm{k}_{1}} /(1-\alpha)<\lambda /(1-\lambda)
$$

Let $\beta_{1}=\lambda+\alpha^{k_{1}}(1-\lambda), \quad \beta_{2}=\lambda-\frac{\alpha^{k_{1}+1}}{1-\alpha}(1-\lambda), \bar{x}=y+\beta_{1} \eta(x, y), \bar{y}=y+\beta_{2} \eta(x, y)$.

Then

$$
0 \leq \beta_{2} \leq \lambda \leq \beta_{1} \leq 1, \lambda=\alpha \beta_{1}+(1-\alpha) \beta_{2}
$$

Thus from Condition C,we have

$$
\begin{aligned}
z+\alpha^{\mathrm{k}_{1}} \eta(x, z) & =y+\lambda \eta(x, y)+\alpha^{k_{1}} \eta(x, y+\lambda \eta(x, y)) \\
& =y+\left[\lambda+\alpha^{k_{1}}(1-\lambda)\right] \eta(x, y) \\
& =y+\beta_{1} \eta(x, y)=\bar{x} .
\end{aligned}
$$

From (2.12)(2.13), we obtain

$$
f(\bar{x})=f\left(z+\alpha^{k_{1}} \eta(x, z)\right)=f\left(z_{k_{1}}\right)<f(z) .
$$

There are two cases to be considered.

(i.) $f(\bar{x}) \geq f(\bar{y})$.It follows from Condition $\mathrm{C}$ that

$$
\begin{aligned}
\bar{y}+\alpha \eta(\bar{x}, \bar{y}) & =y+\beta_{2} \eta(x, y)+\alpha \eta\left(y+\beta_{1} \eta(x, y), y+\beta_{2} \eta(x, y)\right) \\
& =y+\beta_{2} \eta(x, y)+\alpha\left(\beta_{1}-\beta_{2}\right) \eta(x, y)
\end{aligned}
$$




$$
=y+\left[\alpha \beta_{1}+(1-\alpha) \beta_{2}\right] \eta(x, y)=y+\lambda \eta(x, y)=z
$$

Since $f$ is preinvex , this implies

$$
f(z) \leq \alpha f(\bar{x})+(1-\alpha) f(\bar{y}) \leq f(\bar{x}) \text {.which contradicts inequality (2.14). }
$$

(ii.) $f(\bar{x})<f(\bar{y})$. Since $\bar{y}+\alpha \eta(\bar{x}, \bar{y})=y+\lambda \eta(x, y)=z$

By (2.8), we get

$$
f(z)<\alpha f(\bar{x})+(1-\alpha) f(\bar{y}) .
$$

Again, $\bar{x}=y+\beta_{1} \eta(x, y), \bar{y}=y+\beta_{2} \eta(x, y)$ and $f$ is preinvex, we have

$$
\begin{aligned}
& f(\bar{x}) \leq \beta_{1} f(x)+\left(1-\beta_{1}\right) f(y) . \\
& f(\bar{y}) \leq \beta_{2} f(x)+\left(1-\beta_{2}\right) f(y) .
\end{aligned}
$$

According to (2.15)-(2.17), we obtain

$$
f(z)<\lambda f(x)+(1-\lambda) f(y),
$$

Which contradicts (2.11). This completes the proof.

Let the problem of minimum $f(x)$ subject to $x \in K$ be denoted by (P). Now, we can discuss an application of preinvex functions to the problem (P).

Theorem 2.4 Let $K \subseteq R^{n}$ be a nonempty invex set with respect to $\eta: R^{n} \times R^{n} \rightarrow R^{n}$ and $f: K \rightarrow R$ be a preinvex function with respect to $\eta$.If $\bar{x}$ is a local minimum to the problem (P), then $\bar{x}$ is a global one.

Proof. If $\bar{x}$ is a local minimum to the problem (P), then there exists a neighborhood $U \in R^{n}$ with $\bar{x} \in U$ and

$$
f(\bar{x}) \leq f(x), \forall x \in K \cap U
$$

Assume that $\bar{x}$ is not a global one of $(P)$,then there exists $\hat{x} \in K$ with

$$
f(\hat{x})<f(\bar{x})
$$

Since $K \subseteq R^{n}$ be a nonempty invex set with respect to $\eta: R^{n} \times R^{n} \rightarrow R^{n}$ and $f: K \rightarrow R$ be a preinvex function, then $\forall \lambda \in(0,1)$

$$
f(\bar{x}+\lambda \eta(\hat{x}, \bar{x})) \leq \lambda f(\hat{x})+(1-\lambda) f(\bar{x})<f(\bar{x})
$$

i.e.,for any $\forall \lambda \in(0,1)$, we have

$$
f(\bar{x}+\lambda \eta(\hat{x}, \bar{x}))<f(\bar{x})
$$

$\lim _{\lambda \rightarrow 0}(\bar{x}+\lambda \eta(\hat{x}, \bar{x}))=\bar{x}$, so there exists a $\delta \quad(0<\delta<1)$ and for all $\lambda \in(0, \delta)$ with $\bar{x}+\lambda \eta(\hat{x}, \bar{x}) \in K \cap U$. Whic is a contradiction to (2.18). This completes the proof.

Remark 2.2 From Theorem 2.4, we can conclude that preinvex functions constitutes an important class of generalized convex functions in mathematical programming.

\section{Acknowledgment}

The authors also would like to thank two anonymous referees very much for their valuable comments and suggestions, which helped to improve the paper. This work was supported by Natural Science Foundation Project of CQ CSTC (2008BB0346, 2009BB2375), the Foundation Project of ChongQing Educational Committee (KJ080404) and the Science Project Research Foundation of Chongqing Jiao Tong University.

\section{References}

Long X. J. ,Peng Z. Y. and Zeng B. (2009). Remark on cone semistrictly preinvex functions, Optim Lett., 3,337-345.

Mohan, S, R and Neogy, S, K. (1995). On Invex sets and Preinvex Functions. J Math Anal Appl,189, 901-908.

Peng J W and Yang, X, M. (2005). Two Properties of Strictly Preinvex Functions. OR Transactions, 9, 37:42. 
Peng, Z. Y. and Chen, Guo. (2007). Remarks on Prequasi-invex Functions. Journal of China Three Gorges University (Natural Science edition), 6, 70-72.

Peng, Z. Y. and Luo, H. L. (2006). Technical Note on Characterrizations of Strongly Preinvex Functions. Journal of Chongqing Normal University(Natural Science edition), 23, 36--39.(in Chinese).

Weir, T and Mond, B. (1988). Preinvex Functions in Multiple Objective Optimization. J Math Anal Appl.,136, $29-38$.

Yang, X, M and Li, D. (2001). On Properties of Preinvex Functions. J Math Anal Appl, 256, 29-241.

Yang, X, M and Li, D. (2001). Semistrictly Preinvex Functions. J Math Anal Appl, 258, 287-308.

Yang, X, M,Yang, X, Q and Teo, K, L. (2003). Generalized invexity and invariant monotonicicy. J Optim Theory Appl., 117,607-625.

Yang, X, M. (1994). Semistrictly convex Functions. Opsearch, 31, 15-27. 